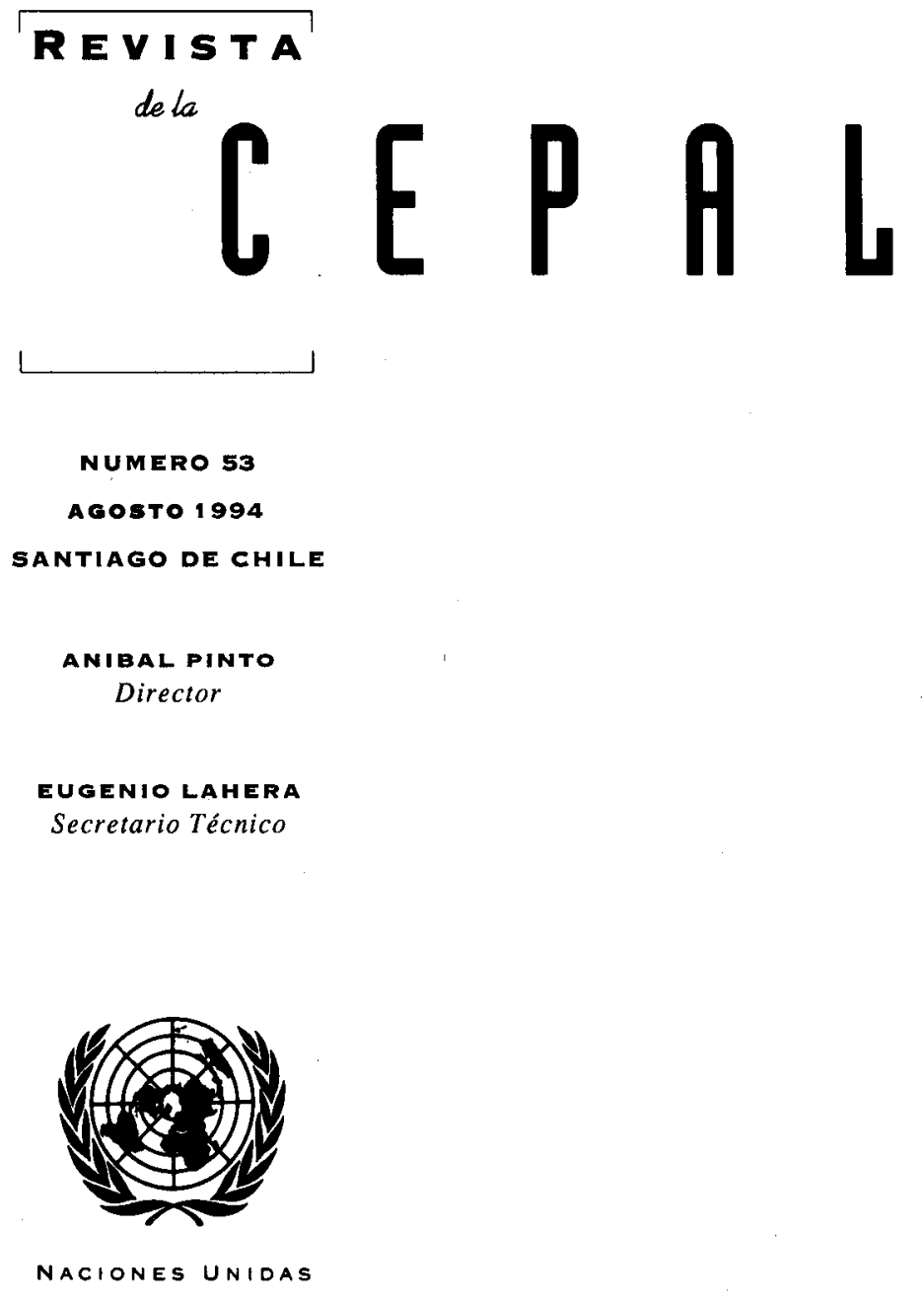


América Latina y el Caribe frente a la economía mundial

Gert Rosenthal

Afluencia de capitales externos y políticas macroeconómicas

Andras Uthoff y Daniel Titelman

Represión fínanciera y patrón de financiamlento latínoamericano

31

Marcos Antonio Macedo Cintra

Políticas de competitividad

49

Wilson Peres

Política industrial y fomento de la competitividad

Osvaldo Rosales

El regionalismo abierto y la integración económica

Juan A. Fuentes $K$.

Transformaciones del trabajo femenino urbano

91

Irma Arriagada

La gestión del agua y las cuencas en América Latina

Axel Dourojeanni

Políticas públicas y competitividad de las exportaciones

agrícolas

Milton von Hesse

Agroindustria y transformación productiva de la pequeña agricultura

Alejandro Schejtman

Grupos privados nacionales en México, 1988-1993

Celso Garrido

Evolución y perspectivas de la reforma y la apertura en China 


\begin{tabular}{|l|l|l|}
\cline { 2 - 3 } & 177 \\
\hline
\end{tabular}

\section{Evolución y perspectivas de la reforma y la apertura en China}

\section{Li Cong}

Economista, Editor Jefe de World Economy and China, Instituto de Estudios Económicos y Políticos Mundiales de la Academia de Ciencias Sociales de China.
En este artículo, escrito especialmente para la Revista de la

CEPAL, se analizan los antecedentes y las perspectivas de la reforma económica de China y de su apertura a la economía internacional. En la sección I se describe el proceso de reforma y apertura de los últimos 15 años y se analizan sus diversos resultados. En la sección II se consideran algunas lecciones de la experiencia china, incluyendo aspectos políticos y sociales además de los propiamente económicos. En la sección III se consideran diversas perspectivas de la economía china hacia finales del presente siglo, mientras en la sección IV y final se analizan tres interrogantes: ¿Es reversible la reforma en China? ¿Se establecerá una economía socialista de mercado en China? ¿Llegará China a ser una superpotencia? 


\section{I}

\section{La reforma y la apertura económicas}

\section{de los últimos 15 años}

En la actualidad, casi todos los países del mundo llevan a cabo algún tipo de reforma y reajuste económicos. Todos se orientan hacia el mundo y tratan de vincular más estrechamente el mercado interno con el mercado mundial. El proceso de reforma y apertura de China presenta similitudes y diferencias con el de otros países.

En este país el proceso de reforma es una transformación de la antigua economía centralmente planificada en una economía socialista de mercado. Por consiguiente, la reforma se realiza con arreglo a la exigencia fundamental de una orientación socialista, lo que representa un autoperfeccionamiento del socialismo con miras a emancipar y desarrollar la fuerza productiva, acelerar el desarrollo económico y el mejoramiento de las condiciones de vida del pueblo y transformar a China en un país moderno, próspero, democrático y altamente civilizado.

China es un país en desarrollo. En comparación con la mayoría de los demás países en desarrollo, aparte la diferencia de sistema social, ella se halla en gran medida en la misma etapa de desarrollo y en el mismo nivel de desarrollo económico. Su reforma económica apunta a construir una economía de mercado y en ello radica la semejanza con muchos países en desarrollo. Pero China se propone construir una economía de mercado socialista y esa es su singularidad

En el pasado, China practicó un sistema altamente centralizado de economía planificada, que era el mismo que tenían la ex Unión de Repúblicas Socialistas Soviéticas y los países de Europa oriental. Después del drástico cambio político acaecido, la antigua Unión Soviética y los países de Europa oriental han abandonado el socialismo y han optado por la privatización y la economía de libre mercado de tipo occidental, mientras que China todavía insiste en el socialismo. Esta es una diferencia fundamental.

Desde el tercer período de sesiones plenarias del XI Congreso del Partido Comunista de China, a finales de 1979, que marcó el comienzo del proceso de reforma y apertura al mundo, China ha pasado por cuatro períodos en 15 años:
1. El periodo inicial de la reforma (1979-1983). En este lapso, la reforma en China estuvo enfocada a las zonas rurales. El país tiene una población rural de 800 millones de personas y la agricultura es el fundamento de la economía nacional. Por lo tanto, es importante que la reforma se inicie en el área rural para estimular el entusiasmo de todos los campesinos en la producción, y acelerar el desarrollo agrícola mediante la supresión de las comunas del pueblo y su reemplazo por un sistema de responsabilidad familiar. Con arreglo a este sistema, la tierra y la producción se asignan por contrato a las familias campesinas y son los propios campesinos quienes deciden lo que habrán de producir y la manera de hacerlo. Este sistema ha despertado en gran medida el entusiasmo y la iniciativa de los campesinos en la producción. Como resultado, se produjo un rápido crecimiento de la producción agropecuaria y una mejora significativa de las condiciones de vida del campesinado. En materia de apertura al mundo, se establecieron durante este período cuatro zonas económicas especiales (de Shenzhen y otras).

2. El periodo de expansión de la reforma (19841988). Sobre la base del progreso alcanzado en la reforma de la agricultura, el enfoque de la reforma se desplazó luego hacia las ciudades. La reforma económica en las ciudades es mucho más complicada que en las zonas rurales, porque supone cambios estructurales en materia de planificación, finanzas, tributación, precios, mano de obra, salarios, etc. Adoptamos el principio de avance gradual, aprovechando las experiencias y enseñanzas del proceso en la práctica. Como resultado de este principio, se logró un progreso adecuado y la economía nacional mostró un rápido crecimiento. China se abrió más ampliamente al mundo: además de las cuatro zonas económicas especiales, Shanghai y 14 ciudades del litoral se convirtieron en ciudades abiertas durante este período.

3. El período de avance tortuoso de la reforma (1988-1991). Como resultado de la insuficiencia de los esfuerzos desplegados por el gobierno en materia de fiscalización y regulación en el plano global, 
se produjo una inversión excesiva en la construcción de bienes de capital, un incremento exagerado del consumo social, un desequilibrio entre la demanda social agregada y la oferta social agregada, un recalentamiento de la economía y un aumento de la tasa de inflación. Para salir de esta situación, se pusieron en práctica medidas de regulación y ajuste y se adoptó una política económica de contracción en estos tres años, mientras se acentuaba aún más la apertura. A las cuatro zonas económicas especiales existentes se sumó la isla de Hainan, que se convirtió en la nueva zona económica especial de mayor tamaño.

4. Finalmente, a partir de 1991 se ha aplicado con éxito una política de regulación y ajuste y la reforma económica, la apertura y el desarrollo económico han entrado en una nueva fase. Vale la pena señalar que Deng Xiaoping sostuvo conversaciones importantes durante su gira de inspección a China meridional y pidió al pueblo que aprovechara el tiempo y emancipara aún más su mente para acelerar el proceso de reforma y apertura. Este llamado estimuló mucho al pueblo en todo el país. En 1992, el XIV Congreso del Partido Comunista de China se planteó la tarea de construir una economía de mercado socialista, lo que aclaró más aún la orientación de la reforma. En este período, el país incrementó más todavía su apertura al mundo. El distrito Pudong de Shanghai ha comenzado a transformarse en una nueva zona de apertura al mundo, y muchas ciudades grandes y medianas a lo largo del río Yan-Tse y las zonas fronterizas, así como todas las capitales provinciales, se han sumado a la condición de ciudades abiertas.

Quince años de reforma y apertura han cambiado profundamente el panorama económico de China:

i) La estructura de propiedad monotípica del pasado ha sido reemplazada por un nuevo modelo de desarrollo conjunto de elementos multieconómicos, en el que la propiedad pública es el fundamento. La participación de la propiedad pública en el producto nacional bruto bajó de $98 \%$ en 1979 a $85 \%$ en 1992 , dentro de la cual el porcentaje correspondiente a la propiedad estatal disminuyó de 55\%-60\% a 35\%-40\% y el de la economía colectiva aumentó considerablemente. Al mismo tiempo, la economía privada y el capital extranjero representaron entre $10 \%$ y $15 \%$ de la economía nacional china.

ii) En el pasado, todas las vinculaciones de las actividades económicas estaban bajo el control y la gestión del plan estatal. Actualmente, las que están bajo el control del plan estatal sólo representan una pequeña parte del PIB; la mayoría abrumadora del resto es regulada por el mercado. Por ejemplo, en 1992 los precios del $80 \%$ de todos los productos básicos estaban regulados por el mercado.

iii) Se han producido cambios considerables en las estructuras financiera, tributaria, de los precios, de la mano de obra, de los salarios, etc.

iv) Se ha conformado un modelo general de apertura al mundo, compuesto por las zonas económicas especiales, las ciudades costeras, las ciudades a lo largo del río Yan-Tse y las zonas fronterizas, así como las capitales provinciales. También se han reformado adecuadamente el comercio exterior y el sistema cambiario.

El proceso de reforma y apertura ha fomentado el desarrollo económico y mejorado las condiciones de vida de la población:

i) De 1979 a 1992, la tasa media de crecimiento anual del PIB fue de $9 \%$; la agricultura creció $5.9 \%$ y la industria $13.2 \%$ por año. La economía china se fortaleció de manera importante.

ii) El comercio exterior evoluciona muy rápidamente. En el período 1979-1992 el incremento anual medio del valor del comercio exterior fue de $16 \%$. En 1992 el valor total de las importaciones y exportaciones ascendió a 165610 millones de dólares. La participación del comercio exterior en el PIB aumentó de $10 \%$ en 1978 a $40 \%$ en 1992. La inversión extranjera mostró un crecimiento acelerado. Hasta ahora se han establecido decenas de miles de empresas mixtas, de asociaciones cooperativas y empresas de propiedad exclusivamente extranjera, $y$ ha ingresado capital extranjero por más de 80 mil millones de dólares, que ha sido invertido.

iii) Se han producido cambios visibles en la participación de las industrias primarias, secundarias y terciarias en el PIB. En 1979 esa participación era de $28.4 \%, 48.6 \%$ y $23.0 \%$ respectivamente, y en 1992 fue de $23.9 \%, 48.2 \%$ y $27.9 \%$.

iv) El ingreso de la población aumentó considerablemente. En el período 1979-1992 la tasa media de crecimiento anual de los salarios de los trabajadores y empleados alcanzó al 7.4\% y el incremento anual medio del nivel de consumo de la población fue de $6.8 \%$. 


\section{II}

\section{Experiencias y problemas}

El proceso de reforma y apertura de China ha obtenido grandes logros. A continuación se resumen algunas experiencias:

i) La estabilidad política y económica es una importante garantía política para el proceso de reforma y apertura y para el desarrollo económico. Huelga decir que la reforma y la apertura no pueden tener éxito en un contexto de inestabilidad política y perturbaciones sociales.

ii) La teoría puede ir de la mano con la práctica. En China, el proceso de reforma y apertura al mundo constituye un hecho sin precedente: no hay experiencia previa ni teoría preconcebida que aplicar. Lo único que podíamos hacer era avanzar primero, reunir las experiencias y enseñanzas del proceso de investigación y ensayo, y luego formular la teoría. Con el progreso de la reforma y la apertura, la teoría que surge de esta gran práctica, madura y se sistematiza gradualmente, y se incorpora en forma concentrada en la teoría del socialismo con características chinas expuesta por Deng Xiaoping. Esta es la brújula que guía la reforma y la apertura hacia la victoria.

iii) La reforma ha avanzado paso a paso, de manera gradual; tuvo lugar primero en las zonas rurales y luego se extendió a las ciudades. No adoptamos una drástica "terapia de choque". La apertura al mundo también se amplió gradualmente. Este avance paulatino del proceso de reforma contribuyó a proteger la economía de las fluctuaciones drásticas y de la confusión, y es una garantía de que no se excederá el límite de sustentabilidad social. De este modo se asegura el desarrollo sustentable de la sociedad.

iv) Las grandes masas de la población deberán beneficiarse con la reforma. Esta es la gran causa de todo el pueblo, cuyo entusiasmo es preciso despertar. Por lo tanto, además de dar a conocer la reforma y de educar al pueblo para ella, es importante que éste obtenga beneficios materiales visibles del proceso de reforma y apertura y que sus condiciones de vida mejoren gradualmente a medida que él avanza. Esto no significa necesariamente, por supuesto, que gente de todos los estratos y de todos los oficios disfrutarán de un aumento por igual en el nivel de vida. China alienta a parte de la población a que haga dinero primero y que reconozca las diferencias en materia de ingreso social, y al mismo tiempo garantiza a toda la población que sus condiciones de vida pueden mejorar hasta cierto punto y acercarse gradualmente al objetivo de prosperidad para todos.

v) Habrá que esforzarse por lograr un avance sincrónico de la reforma y el desarrollo, de manera que la reforma pueda fomentar el desarrollo, y el desarrollo económico y social pueda garantizar la profundización gradual de la reforma. La experiencia china indica que, aun con un régimen socialista, el desarrollo económico no será un proceso estable; las fluctuaciones son inevitables. Pero estamos empeñados en evitar vaivenes bruscos de la economía y en lograr un desarrollo rápido, sólido y sustentable.

Creemos que lo que hemos hecho se ajusta a la realidad del país y a la ley objetiva de reforma, apertura y desarrollo económico. Ello explica justamente por qué China puede lograr un gran éxito en su proceso de reforma y apertura.

Por otra parte, percibimos claramente que subsisten no pocos problemas y dificultades, entre los cuales los principales son:

i) La reforma fomenta un rápido desarrollo económico, pero la eficiencia económica todavía no es elevada, especialmente en las grandes y medianas empresas estatales, la tercera parte de las cuales ha experimentado pérdidas reiteradas. En ellas se tiende a privilegiar exclusivamente la rapidez y no se presta suficiente atención a la eficiencia.

ii) Hay problemas en la estructura industrial, principalmente en las siguientes esferas: la producción agrícola, aunque hizo progresos notables, no ha crecido rápidamente en los últimos años; la industria terciaria marcha a la zaga; y la infraestructura en materia de energía, transporte, correos y telecomunicaciones, etc., no puede satisfacer las exigencias del desarrollo económico y de las condiciones de vida de la población.

iii) Es necesario lograr un equilibrio general entre la demanda social agregada y la oferta social agregada para garantizar un desarrollo económico sustentable al abrigo de fluctuaciones drásticas. Pero 
debido a la preocupación por la velocidad del crecimiento, la demanda social agregada tiende a expandirse de manera arbitraria, especialmente en el caso del crecimiento excesivo de las inversiones en la construcción de bienes de capital, lo que se ha traducido en un recalentamiento de la economía, una mayor presión inflacionaria y un más rápido aumento de los precios.

iv) China es un país de gran extensión y las condiciones de las distintas regiones varían considerablemente. En consecuencia, se hace inevitable un desarrollo desigual de las regiones. Es importante que las áreas relativamente desarrolladas — por ejemplo, la zona costera de China oriental- arrastren consigo a las de menor desarrollo, a fin de reducir gradualmente los desniveles regionales y lograr un desarrollo conjunto.

v) La estrategia de China se centra en la construcción económica y el desarrollo de la fuerza de producción. Esto es indudablemente correcto. Pero es preciso mantener el equilibrio entre desarrollo económico y desarrollo sociocultural. Por ejemplo, la educación todavía está atrasada, no obstante el gran progreso realizado, y el aporte de la ciencia y la tecnología aún es muy insuficiente.

Los problemas y difícultades mencionados son inherentes al proceso de desarrollo de China y tendrán que ser resueltos cuando la reforma se profundice. Naturalmente, no desaparecerán por sí solos sin la adopción de medidas eficaces.

\section{III}

\section{Las perspectivas hacia fines del siglo $\mathrm{XX}$}

El proceso de reforma y apertura ha avanzado considerablemente y ha alcanzado logros significativos en los últimos 15 años, pero aún queda mucha distancia por recorrer para llegar a nuestro objetivo de transformar las viejas estructuras y construir la nueva estructura de una economía socialista de mercado. A fin de realizar esta tarea histórica, debemos hacer grandes esfuerzos por proseguir con las reformas y la apertura y resolver una serie de tareas fundamentales que enfrentamos, a saber:

i) La transformación del mecanismo operacional de las grandes y medianas empresas estatales y el establecimiento de un sistema de empresas modernas. Esto no es sólo una manera básica de modificar la situación de baja eficiencia de dichas empresas, sino también el eslabón principal para construir la estructura de una economía socialista de mercado. Con esa finalidad, es indispensable separar la propiedad de las empresas estatales de su administración e impulsarlas a que ingresen al mercado para lograr su gestión autónoma. El experimento de participación accionaria que se realiza en la actualidad es parte del esfuerzo en esa dirección.

ii) El establecimiento de un sólido sistema de mercado. El mercado se ha desarrollado gradualmente en China durante los 15 años de reforma. Pero hasta el momento, es principalmente el mercado de bienes de consumo el que ha logrado un desarrollo significativo, mientras los mercados de medios de producción, capitales, valores negociables, moneda, tecnología, servicios e información, etc., se hallan aún en su etapa inicial e inclusive algunos de ellos en período de ensayo. Se requieren grandes esfuerzos para acelerar su transición a un sistema de mercado sólido, unificado y abierto.

iii) La transformación completa de la función del Estado. En el pasado, con arreglo al sistema de economía planificada, el gobierno ejercía el control directo de la economía a través del plan estatal (plan principalmente obligatorio) y los medios administrativos. En virtud de una economía socialista de mercado, la función del Estado debe transformarse y pasar de la fiscalización directa de la economía al control y regulación indirectos de ella. Con este fin, es preciso, en primer lugar, cambiar por completo el concepto de Estado, en tanto que éste debe adquirir los medios y la capacidad para ejercer el control y la regulación.

iv) Una mayor apertura al mundo. Además de las zonas económicas especiales, las ciudades costeras, las ciudades a lo largo del río Yan-Tse y las zonas fronterizas, así como las capitales provinciales, es necesario abrir las ciudades que se hallan a lo largo de las principales líneas férreas y carreteras. Más importante aún es la reforma estructural del comercio exterior, para que las normas y reglamentaciones del comercio exterior de China se ajusten gradualmente a 
los convenios del mercado mundial, disminuyan los aranceles y se liberalicen las importaciones y exportaciones. Se hará lo posible por lograr cuanto antes el restablecimiento de la participación de China en el GATT. Se mejorará todavía más el tratamiento de las inversiones para atraer más capital extranjero, y se fortalecerá más aún la cooperación económica con todo el mundo.

En noviembre de 1993, en el tercer período de sesiones plenarias del XIV Congreso del Comité Central del Partido Comunista de China, se adoptó una decisión acerca de varios asuntos relativos al establecimiento de un sistema de economía socialista de mercado, y se señaló que la reforma y apertura de nuestro país y la causa de la construcción de la modernidad entraban a una nueva etapa. El nuevo sistema económico deberá establecerse primordialmente a fines del presente siglo. La decisión establece una serie de principios, políticas y medidas para resolver varios problemas complejos. Se prevé que la reforma y apertura de China avanzarán incluso con mayor rapidez y vigor en los próximos años.

Al mismo tiempo, la construcción económica en China adquirirá mayor impulso. El objetivo estratégico del país es el de duplicar a fines del presente siglo su PNB de los años ochenta, que a su vez había duplicado el del decenio anterior; en ese lapso las condiciones de vida de la población pueden alcanzar un nivel decente, no muy alto todavía, pero libre de pobreza. No sólo se satisfarán sus necesidades de alimentación y vestuario, sino que también disfrutarán de una mejoría considerable en su nivel de vida. El nivel educacional y cultural se elevará considerablemente. Hay posibilidades amplias de que este objetivo estratégico se logre antes de lo programado.

\section{IV}

\section{Respuestas a algunas interrogantes}

Conviene entender correctamente algunos puntos relacionados con el proceso de reforma y apertura en China.

¿Deberá revertirse o abandonarse el proceso de reforma y apertura de China? Debemos decir que esto es absolutamente imposible, porque la construcción del socialismo con características chinas mediante un proceso de reforma y apertura al mundo es la única vía correcta hacia la felicidad, lo que se comprendió finalmente después de prolongadas exploraciones y reiterados reveses y sufrimientos desde la fundación de la República Popular de China. Este punto concita consenso nacional. El pueblo chino de todas las nacionalidades tiene interés vital en él. Nunca podrá cambiar.

Quince años de reforma y apertura han modificado considerablemente el panorama de este país y han mejorado las condiciones de vida de las grandes masas de la población. El proceso de reforma y apertura cuenta con el apoyo unánime del pueblo chino.

El proceso de reforma y apertura está guiado por la teoría de la construcción del socialismo con características chinas avanzada por Deng Xiaoping, lo que se ha institucionalizado en la Constitución de China.

¿Podrá China establecer en el futuro un sistema de economía socialista de mercado? Esta es una pre- gunta que interesa a muchos amigos. Algunos piensan que el socialismo no puede combinarse con el mercado. De hecho, esto es lo que muchos de nosotros creímos durante mucho tiempo. Pensábamos que el socialismo sólo podría adoptar una economía planificada y que no debía desarrollar una economía de mercado. Después de exploraciones por más de diez años, este asunto ha sido aclarado fundamentalmente en la teoría y en la práctica en China. Ahora comprendemos que el socialismo es un sistema social básico, en tanto que la planificación y el mercado son medios o mecanismos diferentes para desarrollar y reglamentar la economía, y pueden ser utilizados por el capitalismo y también por el socialismo. En el pasado creíamos sólo en el uso del mecanismo de planificación, especialmente la planificación obligatoria, y rechazábamos los mecanismos del mercado. Esta es una concepción sumamente unilateral, que nos causó grandes sufrimientos. Actualmente tenemos una nueva concepción y más confianza en nuestras acciones.

¿Se convertirá China en una superpotencia? En los últimos años ha habido reacciones en este sentido en muchos países frente al rápido crecimiento de la economía china: se ha dicho que China ya se transformó en una gran potencia, o que llegará a serlo en 
el próximo siglo; que su poderío económico ha ascendido al tercer lugar en el mundo, siendo superado sólo por el de los Estados Unidos y del Japón; que China es actualmente uno de los "polos" del mundo. Algunos incluso se preocupan de que pueda convertirse en una amenaza para los demás cuando se vuelva más poderosa. Esas hipótesis carecen de fundamento. Alguien las formuló por motivos ocultos, para inducir a la gente a error y crear suspicacias contra nuestro país.

Debemos señalar que aunque China ha alcanzado grandes éxitos en su reforma económica, y logros sobresalientes en su desarrollo económico, y que alcanzará mayores éxitos y logros en el futuro, será siempre un factor de paz y estabilidad en el mundo y nunca lo contrario. Y ello debido a que:

i) China es un país con una enorme población. Aunque su importancia económica es bastante grande, su ingreso por habitante todavía no es elevado. China aún es un país en desarrollo e incluso entre los países en desarrollo su nivel económico ocupa un lugar de retaguardia; y deberá realizar ingentes esfuerzos durante mucho tiempo antes de que pueda alcanzar el nivel de un país próspero y rico. En consecuencia, enfocará decididamente sus esfuerzos hacia la construcción económica, y nunca será una amenaza para otros, aun cuando tenga más poderío en el futuro.

ii) Durante muchos años, China tuvo una historia amarga y humillante como país oprimido y agredido. Siempre siente simpatía y solidaridad por la causa justa de países y naciones más débiles, y nunca será agresora y opresora de otros países cuando se haga más poderosa.

iii) China persiste en observar los cinco principios de la coexistencia pacífica y sigue una línea diplomática de independencia, autonomía y paz. Guiada por esos principios y ese lineamiento, ha mejorado constantemente sus relaciones con los demás países y se ha ganado el respeto y la confianza de los pueblos del mundo.

(Traducido del inglés.) 